\title{
Study of Organisms Causing Osteomyelitis in a Tertiary Care Hospital
}

\author{
Rupali Bhaurao Meshram ${ }^{1}$, Sunita Raj Gajbhiye², Sharmila Sanjay Raut ${ }^{3}$ \\ ${ }^{1}$ Department of Microbiology, Indira Gandhi Government Medical College, Nagpur, Maharashtra, India. \\ ${ }^{2}$ Department of Microbiology, Indira Gandhi Government Medical College, Nagpur, Maharashtra, India. \\ ${ }^{3}$ Department of Microbiology, Indira Gandhi Government Medical College, Nagpur, Maharashtra, India.
}

\section{ABSTRACT}

\section{BACKGROUND}

The term osteomyelitis (OSM) was first used by the French surgeon Edouard Chassaignac in 1852, who defined the disease as an inflammatory process accompanied by bone destruction caused by an infecting microorganism. The clinical manifestation and the natural history of OSM depend on several factors. OSM mostly affects the growing ends of long bones. We wanted to study the organisms causing osteomyelitis and their antimicrobial susceptibility pattern.

\section{METHODS}

Pus and bone aspirate were collected from 115 diagnosed patients of osteomyelitis and were processed for isolation of organisms by standard microbiological techniques. Isolates were identified by various biochemical reactions and were subjected to antimicrobial susceptibility test as per CLSI guidelines by Kirby-Bauer disk diffusion technique on Mueller Hinton agar (MHA). Data collected in the questionnaire was entered and analysed in Epi Info software version 7.2.

\section{RESULTS}

In 101 samples, 116 organisms were isolated. In 14 samples no organism was isolated, which can be attributed to the viral aetiology, parasites and anaerobes. Acute Osteomyelitis (AOSM) was found to be more common in the age group of 1-10 years, whereas chronic osteomyelitis (COSM) was found more commonly in 21-30 and 31-40 years age group. Male to Female ratio was 2.2:1. Bones involved in AOSM and COSM were mostly femur followed by tibia and humerus. S. aureus was the most predominant isolate. All the isolates of $S$. aureus showed $100 \%$ sensitivity to Vancomycin, Amikacin, Netilmicin, Chloramphenicol. Out of 48 isolates of S. aureus, $37.50 \%$ were MRSA, $6.25 \%$ were ICR, $14.58 \%$ were MRSA+ICR found.

\section{CONCLUSIONS}

Osteomyelitis is found to be highest in third decade, with the males being predominantly affected. Acute osteomyelitis is predominantly seen in children, whereas chronic osteomyelitis in adults. Even though Staphylococcus aureus has always remained the most common etiological agent of osteomyelitis, increasing infections due to Gram negative bacilli and even poly-microbial infections are gaining importance. MRSA infection is known to increase post-operative complications. Introduction of MBL or carbapenemase production in Gram negative bacilli is a matter of great concern. Timely knowledge of aetiology and antimicrobial resistance pattern of osteomyelitis isolates can help in rational use of antibiotics and control of drug resistance.

\section{KEY WORDS}

Osteomyelitis, Staphylococcus aureus, MRSA, Long Bones, Males

\author{
Corresponding Author: \\ Dr. Sunita Gajbhiye, \\ Oasis, Plot No. 11 \& 12, \\ Anand Nagar, Atrey Layout, \\ Near Datta Meghe College, \\ Nagpur-440022, Maharashtra, India. \\ E-mail: sunitarajgaj@yahoo.com
}

DOI: $10.14260 /$ jemds $/ 2020 / 8$

Financial or Other Competing Interests: None.

How to Cite This Article:

Meshram RB, Gajbhiye SR, Raut SS. Study of organisms causing osteomyelitis in a tertiary care hospital. J. Evolution Med. Dent. Sci. 2020;9(01):33-37, DOI: 10.14260/jemds/2020/8

Submission 26-10-2019,

Peer Review 20-12-2019,

Acceptance 27-12-2019,

Published 06-01-2020. 


\section{BACKGROUND}

Bone and joint infections are painful for patients and frustrating for them and their doctors. ${ }^{1}$ The term osteomyelitis (OSM) was first used by the French surgeon Edouard Chassaignac in 1852, who defined the disease as an inflammatory process accompanied by bone destruction and caused by an infecting microorganism. ${ }^{2}$ This disease is differentiated according to the aetiology, pathogenesis, and degree of bone involvement, as well as age and the immune condition of the patient. It can involve different structures such as the bone marrow, cortex, periosteum, and parts of the surrounding soft tissues, or remain localized. The clinical manifestation and the natural history of OSM depend on several factors like age of patients, site of infection, virulence of infecting organism and the patients resistance. ${ }^{3}$ OSM mostly affects the growing ends of long bones and it is more common in the lower extremity at metaphysis of femur and proximal end of tibia. ${ }^{4}$ Historically, acute haematogenous osteomyelitis has been described in prepubertal children. It involves mostly the metaphysis of long bones (particularly tibia and femur), in most cases as a single focus. Although rare in adults, it most frequently involves the vertebral bodies. ${ }^{1}$ Osteomyelitis due to local spread from a contiguous contaminated source of infection follows trauma, bone surgery, or joint replacement. It implies an initial infection that gains access to bone. It can occur at any age and can involve any bone. ${ }^{1}$ Historically, osteomyelitis has been categorized as acute, subacute or chronic based on the time of disease onset (i.e., occurrence of infection or injury). The duration of symptoms of infection is in fact associated with peculiar anatomo-pathological findings and clinical and diagnostic features and influences the therapeutic decisions. Acute osteomyelitis is diagnosed within 2 weeks after disease onset, subacute osteomyelitis within one to several months and chronic osteomyelitis after a few months. ${ }^{2}$

Osteomyelitis in children is most often acute and secondary to haematogenous spread. The diagnosis can usually be made from the clinical signs, but requires a high index of suspicion. In neonates and infants, osteomyelitis has certain peculiar features. ${ }^{3}$ The subacute and chronic forms of osteomyelitis usually occur in adults. Generally, these bone infections are secondary to an open wound, most often an open injury to bone and surrounding soft tissues. Localized bone pain, erythema and drainage around the affected area are frequently present. The cardinal signs of subacute and chronic osteomyelitis include draining sinus tracts, deformity, instability and local signs of impaired vascularity, range of motion and neurologic status. ${ }^{2}$ The majority of osteomyelitis cases in adults are generally chronic in nature and are associated with a traumatic insult to the involved area. The diagnosis of osteomyelitis is first suspected on clinical grounds. The most important step, is to isolate the offending organisms so that appropriate antimicrobial therapy can be chosen. ${ }^{1}$

Other conditions that may be confused with OSM are cellulitis, septic arthritis, trauma and bone infarction. Acute osteomyelitis can respond to antibiotic treatment alone. Chronic osteomyelitis is associated with avascular necrosis of bone and formation of sequestrum (dead bone); Surgical debridement is therefore necessary for cure in addition to antibiotic therapy. 1

Thus, because of the changes in the manifestations, epidemiology, and etiological agents, it is important to make a precise microbiological diagnosis. It is important to know microbiological aetiology in different types of osteomyelitis in our region. Early antibiotic treatment, before extensive destruction of bone or necrosis, produces the best results. Hence, the study was done to evaluate the microorganisms causing OSM along with their antimicrobial susceptibility pattern.

\section{METHODS}

The study was carried out in department of Microbiology at a tertiary care hospital in Nagpur region of Central India, over the period of 2 years. Pus and bone aspirate were collected from clinically and radiologically diagnosed 115 patients of osteomyelitis, attending outpatient department and/or admitted to wards of the hospital. Sample size of 115 patients was selected on the basis of previous articles related to our topic and statistician consultation. Data collected in the questionnaire were entered and analysed in Epi Info software version $7.2^{5}$. Categorical data was analysed by means of mean, standard deviation and quantitative data by proportion and percentage. The group differences were tested using chi-square, or others depending on the type of variable. $p$ - value $<0.05$ was considered to derive a level of significance.

Diagnosis was made on the basis of duration, as acute OSM and chronic OSM. Acute OSM characterised by systemic illness, absence of bony radiological changes at presentation, history less than 10 days. Chronic OSM characterised by systemic illness present or absent depending on presentation, Bony radiological changes in MRI and X-ray at presentation, history of previous episode or episodes of infection. Though it is a deep-seated infection we did not include CBC, CRP, ESR in our study, since it was not having any correlation with any parameter and was not significant. Specimens were processed for isolation of aerobic bacteria, Mycobacteria and Fungi by standard Microbiological techniques. ${ }^{6}$ Pus sample was inoculated on the following media ${ }^{6}$ Blood agar (BA), MacConkey agar (MA), Chocolate agar (CA), Sabouraud dextrose agar (SDA), Lowenstein Jensen medium (LJ). Isolates were identified by various biochemical reactions. Each isolate was subjected to antimicrobial susceptibility test as per CLSI guidelines $^{7}$ by Kirby-Bauer disk diffusion technique on Mueller Hinton agar (MHA). ${ }^{8}$ In our study maximum isolates were of Staphylococcus aureus, so MIC of Vancomycin was done by E-strip. We have done disk diffusion test for other antibiotics, since it is recommended for antimicrobial susceptibility test according to CLSI guidelines. Tobramycin is tested for Gram negative organisms and Staphylococcus aureus as a third line drug for deep seated infections according to CLSI guidelines. 


\section{RESULTS}

\begin{tabular}{|c|c|c|c|c|c|c|}
\hline \multirow{2}{*}{ Organism } & \multicolumn{3}{|c|}{ AOSM (n=20) } & \multicolumn{3}{c|}{ COSM (n=96) } \\
\cline { 2 - 7 } & Single & Mixed & Total (\%) & Single & Mixed & Total (\%) \\
\hline S. aureus & 08 & -- & $08(40.00)$ & 40 & -- & $40(41.67)$ \\
\hline S. epidermidis & -- & 01 & $01(5.00)$ & 02 & -- & $02(2.08)$ \\
\hline E. faecium & -- & -- & -- & 02 & -- & $02(2.08)$ \\
\hline K. pneumoniae & 01 & 02 & $03(15.00)$ & 12 & 05 & $17(17.41)$ \\
\hline C. koseri & -- & 01 & $01(5.00)$ & 03 & 03 & $06(6.26)$ \\
\hline E. coli & -- & 01 & $01(5.00)$ & 01 & 03 & $04(4.17)$ \\
\hline S. Typhi & 01 & -- & $01(5.00)$ & 01 & -- & $01(1.04)$ \\
\hline P. mirabilis & -- & 01 & $01(5.00)$ & -- & -- & -- \\
\hline E. cloacae & -- & -- & -- & 01 & -- & $01(1.04)$ \\
\hline A. baumannii & 01 & 02 & $03(15.00)$ & 07 & 03 & $10(10.42)$ \\
\hline P. aeruginosa & 01 & -- & $01(5.00)$ & 03 & 06 & $09(9.38)$ \\
\hline A. Iwoffii & -- & -- & -- & -- & 01 & $01(1.04)$ \\
\hline S. maltophilia & --- & -- & -- & -- & 01 & $01(1.04)$ \\
\hline C. albicans & -- & -- & -- & 02 & -- & $02(1.04)$ \\
\hline Total & \multicolumn{7}{|c|}{ Table 1. Pathogens Isolated Singly and in Mixed Cultures in OSM } \\
\hline \multicolumn{6}{|c|}{}
\end{tabular}

\begin{tabular}{|c|c|c|c|c|c|c|c|c|c|}
\hline \multirow{2}{*}{$\begin{array}{c}\text { Antibiotics/ } \\
\text { Organisms } \\
\text { (N) }\end{array}$} & \multicolumn{9}{|c|}{ Resistance n (\%) } \\
\hline & a & $\sum_{<}^{0}$ & $\sum_{<}^{U}$ & S & X & ¿̦ & 远 & $\sum_{x}^{x}$ & $\sum_{0}$ \\
\hline S. aureus $(48)$ & \begin{tabular}{|c|}
47 \\
$(97.92)$
\end{tabular} & - & - & - & \begin{tabular}{|c|}
25 \\
$(52.08)$
\end{tabular} & - & - & - & - \\
\hline S. epidermidis ${ }^{(3)}$ & $\begin{array}{c}3 \\
(100) \\
\end{array}$ & - & - & - & $\begin{array}{c}1 \\
(33.33) \\
\end{array}$ & - & - & - & - \\
\hline E. faecium ${ }^{(2)}$ & \begin{tabular}{|c|}
2 \\
$(100)$
\end{tabular} & $\begin{array}{c}2 \\
(100)\end{array}$ & - & - & - & - & - & - & - \\
\hline \begin{tabular}{|c|} 
K. pneumoniae \\
(20)
\end{tabular} & - & $\begin{array}{c}20 \\
(100)\end{array}$ & $\begin{array}{c}19 \\
(95)\end{array}$ & $\begin{array}{c}8 \\
(40)\end{array}$ & $\begin{array}{c}15 \\
(75)\end{array}$ & $\begin{array}{c}15 \\
(75)\end{array}$ & $\begin{array}{c}14 \\
(70)\end{array}$ & $\begin{array}{c}17 \\
(85)\end{array}$ & $\begin{array}{c}12 \\
(85)\end{array}$ \\
\hline C. koseri ${ }^{(7)}$ & - & $\begin{array}{c}6 \\
(85.71) \\
\end{array}$ & $\begin{array}{c}7 \\
(100) \\
\end{array}$ & \begin{tabular}{|c|}
6 \\
$(85.71)$ \\
\end{tabular} & \begin{tabular}{|c|}
6 \\
$(85.71)$ \\
\end{tabular} & $\begin{array}{c}6 \\
(85.71) \\
\end{array}$ & $\begin{array}{c}6 \\
(85.71) \\
\end{array}$ & \begin{tabular}{|c|}
6 \\
$(85.71)$ \\
\end{tabular} & $\begin{array}{c}6 \\
(85.71) \\
\end{array}$ \\
\hline E. coli ${ }^{(5)}$ & - & $5(100)$ & $\begin{array}{c}5 \\
(100) \\
\end{array}$ & $\begin{array}{c}3 \\
(60) \\
\end{array}$ & $\begin{array}{c}4 \\
(80)\end{array}$ & $\begin{array}{c}4 \\
(80)\end{array}$ & $\begin{array}{c}4 \\
(80) \\
\end{array}$ & \begin{tabular}{c|}
4 \\
$(80)$
\end{tabular} & $\begin{array}{c}4 \\
(80)\end{array}$ \\
\hline S. Typhi ${ }^{(2)}$ & - & $\begin{array}{c}2 \\
(100)\end{array}$ & $\begin{array}{c}2 \\
(100)\end{array}$ & $\begin{array}{c}1 \\
(50)\end{array}$ & $\begin{array}{c}1 \\
(50)\end{array}$ & $\begin{array}{c}1 \\
(50)\end{array}$ & $\begin{array}{c}1 \\
(50)\end{array}$ & $\begin{array}{c}1 \\
(50)\end{array}$ & $\begin{array}{c}1 \\
(50)\end{array}$ \\
\hline \begin{tabular}{|l|} 
P. mirabilis \\
$(1)$
\end{tabular} & - & $1(100)$ & 00 & 00 & 00 & 00 & 00 & 00 & 00 \\
\hline E. cloacae $^{(1)}$ & - & $\begin{array}{c}1 \\
(100)\end{array}$ & $\begin{array}{c}1 \\
(100)\end{array}$ & 00 & \begin{tabular}{|c|}
1 \\
$(100)$
\end{tabular} & $\begin{array}{c}1 \\
(100)\end{array}$ & $\begin{array}{c}1 \\
(100)\end{array}$ & $\begin{array}{c}1 \\
(100)\end{array}$ & \begin{tabular}{|c|}
1 \\
$(100)$
\end{tabular} \\
\hline \begin{tabular}{|c|} 
A. baumannii \\
$(13)$
\end{tabular} & - & - & - & - & - & $\begin{array}{c}13 \\
(100)\end{array}$ & $\begin{array}{c}13 \\
(100)\end{array}$ & - & $\begin{array}{c}13 \\
(100)\end{array}$ \\
\hline \begin{tabular}{|c|} 
P. aeruginosa \\
$(10)$
\end{tabular} & - & - & - & - & - & $\begin{array}{c}6 \\
(60) \\
\end{array}$ & - & - & $7(70)$ \\
\hline A. lwoffii $(1)$ & - & - & - & - & - & $1(100)$ & $\begin{array}{c}13 \\
(100)\end{array}$ & - & \begin{tabular}{|c|}
13 \\
$(100)$
\end{tabular} \\
\hline S. maltophilia ${ }^{(1)}$ & - & - & - & - & - & $\begin{array}{c}1 \\
(100)\end{array}$ & - & - & - \\
\hline
\end{tabular}

Table 2a. Antimicrobial Resistance Pattern among the Isolates of OSM P- Penicillin, AMP- Ampicillin, AMC- Amoxyclavulanate, CZ- Cefazolin, CX- Cefoxitin, CAZ-Ceftazidime, CTX-Cefotaxime, CXM- Cefuroxime, CPM- Cefipime

\begin{tabular}{|c|c|c|c|c|c|c|c|c|c|c|}
\hline \multirow{2}{*}{$\begin{array}{l}\text { Antibiotics/ } \\
\text { Organisms } \\
\text { (N) }\end{array}$} & \multicolumn{10}{|c|}{ Resistance n (\%) } \\
\hline & $\Sigma$ & 5 & 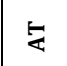 & $\sum_{ٍ}$ & తે & 刭 & $\stackrel{\Xi}{\Xi}$ & 产 & $\stackrel{0}{\circ}$ & $w$ \\
\hline S. aureus ${ }^{(48)}$ & - & - & - & - & - & \begin{tabular}{|c|}
4 \\
$(8.33)$
\end{tabular} & - & 00 & $\begin{array}{c}3 \\
(6.25)\end{array}$ & $\begin{array}{c}17 \\
(35.42)\end{array}$ \\
\hline \begin{tabular}{|c|} 
S. epidermidis \\
${ }_{3}(3)$
\end{tabular} & - & - & - & - & - & \begin{tabular}{|c|}
1 \\
$(33.33)$ \\
\end{tabular} & - & $\begin{array}{c}1 \\
(33.33) \\
\end{array}$ & $\begin{array}{c}1 \\
(33.33) \\
\end{array}$ & 00 \\
\hline E. faecium $^{(2)}$ & - & - & - & - & - & - & $\begin{array}{c}2 \\
(100) \\
\end{array}$ & - & . & \begin{tabular}{|c}
2 \\
$(100)$ \\
\end{tabular} \\
\hline \begin{tabular}{|c|} 
K. pneumoniae \\
$(20)$
\end{tabular} & \begin{tabular}{|c|}
11 \\
$(55)$
\end{tabular} & $\begin{array}{c}6 \\
(30)\end{array}$ & \begin{tabular}{|l|}
14 \\
$(70)$
\end{tabular} & $\begin{array}{l}1 \\
(5)\end{array}$ & - & $\begin{array}{c}6 \\
(30) \\
\end{array}$ & - & $\begin{array}{c}4 \\
(20)\end{array}$ & $\begin{array}{c}5 \\
(25)\end{array}$ & 2 \\
\hline C. koseri (7) & $\begin{array}{c}6 \\
(85.71\end{array}$ & $\begin{array}{c}4 \\
(57.14)\end{array}$ & $\begin{array}{c}6 \\
(85.71)\end{array}$ & $\begin{array}{c}2 \\
(28.57)\end{array}$ & - & $\begin{array}{c}5 \\
(71.43)\end{array}$ & - & $\begin{array}{c}5 \\
(71.43)\end{array}$ & $\begin{array}{c}5 \\
5 \\
(71.43)\end{array}$ & - \\
\hline E. coli ${ }^{(5)}$ & $\begin{array}{c}2 \\
(40) \\
\end{array}$ & \begin{tabular}{|c|}
2 \\
$(40)$
\end{tabular} & \begin{tabular}{|c|}
5 \\
$(100)$ \\
\end{tabular} & $\begin{array}{c}1 \\
(20)\end{array}$ & - & $\begin{array}{c}3 \\
(60) \\
(60)\end{array}$ & - & $\begin{array}{c}2 \\
(40) \\
\end{array}$ & $\begin{array}{c}2 \\
(40) \\
\end{array}$ & - \\
\hline S. Typhi(2) & $\begin{array}{c}1 \\
(50) \\
\end{array}$ & 00 & $\begin{array}{c}1 \\
(50)\end{array}$ & 00 & - & 00 & - & 00 & 00 & . \\
\hline P. mirabilis (1) & 00 & 00 & $\begin{array}{c}1 \\
(100)\end{array}$ & 00 & - & 00 & - & 00 & 00 & - \\
\hline E. cloacae $^{(1)}$ & \begin{tabular}{|c|}
1 \\
$(100)$
\end{tabular} & 00 & $\begin{array}{c}1 \\
(100)\end{array}$ & 00 & - & 00 & - & 00 & $\begin{array}{c}1 \\
(100)\end{array}$ & - \\
\hline \begin{tabular}{|c} 
A. baumannii \\
$(13)$
\end{tabular} & $\begin{array}{c}12 \\
(92.31 \\
\end{array}$ & \begin{tabular}{|c|}
11 \\
$(84.62)$ \\
\end{tabular} & $\begin{array}{c}6 \\
(46.15) \\
\end{array}$ & $\begin{array}{c}5 \\
(38.46) \\
\end{array}$ & - & \begin{tabular}{|c|}
8 \\
$(61.54)$ \\
\end{tabular} & - & $\begin{array}{c}7 \\
(53.85) \\
\end{array}$ & $\begin{array}{c}8 \\
61.54) \\
\end{array}$ & - \\
\hline \begin{tabular}{|c|} 
P. aeruginosa \\
$(10)$
\end{tabular} & \begin{tabular}{|c|}
4 \\
$(40)$
\end{tabular} & \begin{tabular}{|c|}
6 \\
$(60)$
\end{tabular} & $\begin{array}{c}4+.05) \\
(40)\end{array}$ & $\begin{array}{c}0.40) \\
(10)\end{array}$ & $\begin{array}{c}1 \\
(10)\end{array}$ & $6(60)$ & - & \begin{tabular}{|c}
6 \\
$(60)$
\end{tabular} & $\begin{array}{c}5 \\
(50)\end{array}$ & - \\
\hline A. lwoffii ${ }^{(1)}$ & \begin{tabular}{|c|}
1 \\
$(100)$
\end{tabular} & $\begin{array}{c}1 \\
(100)\end{array}$ & $\begin{array}{c}1 \\
(100)\end{array}$ & $\begin{array}{c}1 \\
(100)\end{array}$ & - & 00 & & 00 & 00 & \\
\hline \begin{tabular}{|c} 
S. maltophilia \\
$(1)$
\end{tabular} & & - & $(100)$ & - & - & - & & - & 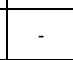 & \\
\hline Table $2 b . A$ & $n+i m$ & $\boldsymbol{o b}$ & Res & $\overline{a n}$ & $a t$ & $n a$ & ongt & $e I$ & tes o & fOSM \\
\hline
\end{tabular}

\begin{tabular}{|c|c|c|c|c|c|c|c|c|c|c|}
\hline \multirow[b]{2}{*}{$\begin{array}{l}\text { Antibiotics/ } \\
\text { Organisms } \\
\text { (N) }\end{array}$} & \multicolumn{10}{|c|}{ Resistance n (\%) } \\
\hline & 愛 & t⿹弋工 & ㅎํ & 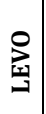 & ชิ & 롭 & 贸 & $\mathbf{I}$ & $\mid \begin{array}{l}z \\
\vdots \\
\mathbf{z}\end{array}$ & 尺े \\
\hline S. aureus ${ }^{(48)}$ & 00 & $\begin{array}{c}6 \\
(12.50)\end{array}$ & \begin{tabular}{|c|}
18 \\
$(37.50)$ \\
\end{tabular} & - & $\begin{array}{c}11 \\
(22.92)\end{array}$ & 00 & \begin{tabular}{|c|}
27 \\
$(56.25)$ \\
\end{tabular} & $\begin{array}{c}1 \\
(2.08)\end{array}$ & $00^{*}$ & \\
\hline S. epidermidis ${ }^{(3)}$ & $\begin{array}{c}1 \\
(33.33) \\
\end{array}$ & $\begin{array}{c}1 \\
(33.33) \\
\end{array}$ & \begin{tabular}{|c|}
2 \\
$(66.67)$ \\
\end{tabular} & - & $\begin{array}{c}1 \\
(33.33) \\
\end{array}$ & 00 & \begin{tabular}{|c|}
3 \\
$(100)$
\end{tabular} & 00 & $00^{\circ}$ & \\
\hline E. faecium $^{(2)}$ & - & $\begin{array}{c}2 \\
(100)\end{array}$ & $\begin{array}{c}2 \\
(100)\end{array}$ & - & - & 00 & $\begin{array}{c}2 \\
(100) \\
\end{array}$ & - & $00^{*}$ & \\
\hline K. pneumoniae ${ }^{(20)}$ & $5(25)$ & $7(35)$ & $4(20)$ & & & $\begin{array}{c}5 \\
(25)\end{array}$ & & & & \\
\hline C. $\operatorname{koseri}^{(7)}$ & $\begin{array}{c}5 \\
(71.43)\end{array}$ & $\begin{array}{c}4 \\
(57.14) \\
\end{array}$ & \begin{tabular}{|c|}
5 \\
$(71.43)$ \\
\end{tabular} & & & $\begin{array}{c}2 \\
28.57) \\
\end{array}$ & & & & \\
\hline E. coli $\left({ }^{(5)}\right.$ & $\begin{array}{c}2 \\
440) \\
\end{array}$ & $\begin{array}{c}3 \\
(60) \\
\end{array}$ & \begin{tabular}{|c|}
4 \\
$(80)$ \\
\end{tabular} & & & $\begin{array}{c}2 \\
(40) \\
\end{array}$ & & & & \\
\hline S. Typhi(2) & 00 & $1(50)$ & \begin{tabular}{|c|}
2 \\
$(100)$ \\
\end{tabular} & & & 00 & & & & \\
\hline P. mirabilis ${ }^{(1)}$ & 00 & $1(100)$ & \begin{tabular}{c|}
1 \\
$(100)$ \\
\end{tabular} & & & 00 & & & & \\
\hline E. cloacae ${ }^{(1)}$ & $\begin{array}{c}1 \\
(100) \\
(100\end{array}$ & 00 & 00 & & & 00 & & & & \\
\hline A. baumannii ${ }^{(13)}$ & - & - & \begin{tabular}{|c|}
8 \\
$(61.54)$ \\
\end{tabular} & - & - & - & - & - & - & 00 \\
\hline P. aeruginosa ${ }^{(10)}$ & $\begin{array}{c}5 \\
(50) \\
\end{array}$ & - & \begin{tabular}{|c|}
4 \\
$(40)$ \\
\end{tabular} & - & - & - & - & - & - & 00 \\
\hline A. Iwoffii (1) & - & - & 00 & - & - & - & - & - & - & 00 \\
\hline S. maltophilia ${ }^{(1)}$ & - & - & - & 00 & - & 00 & - & - & - & - \\
\hline
\end{tabular}

Vancomycin MIC was performed by E-strip

Table 2c. Antimicrobial Resistance Pattern among the Isolates of OSM

NET- Netilmicin, TET- Tetracycline, CIP- Ciprofloxacin, LEVO- Levofloxacin, CD-

Clindamycin, CHL- Chloramphenicol, RIF- Rifampicin, LZ- Linezolid, VAN-

Vancomycin, PB- Polymyxin B

\begin{tabular}{|c|c|c|c|c|c|}
\hline Organisms & $\begin{array}{c}\text { No. of } \\
\text { Isolates }\end{array}$ & $\begin{array}{c}\text { ESBL } \\
\text { Producer } \\
(\mathbf{\%})\end{array}$ & $\begin{array}{c}\text { AmpC } \\
\text { Producer } \\
(\mathbf{\%})\end{array}$ & $\begin{array}{c}\text { ESBL + } \\
\text { AmpC } \\
\mathbf{( \% )}\end{array}$ & $\begin{array}{c}\text { MBL } \\
\text { Producer } \\
(\mathbf{\%})\end{array}$ \\
\hline K. pneumoniae & 20 & $04(20)$ & $03(15)$ & $03(15)$ & 00 \\
\hline C. koseri & 07 & $01(14.29)$ & 00 & 00 & $01(14.29)$ \\
\hline E. coli & 05 & $02(40)$ & 00 & 00 & $01(20)$ \\
\hline S. Typhi & 02 & 00 & 00 & 00 & 00 \\
\hline P. mirabilis & 01 & 00 & 00 & 00 & 00 \\
\hline E. cloacae & 01 & 00 & $01(100)$ & 00 & 00 \\
\hline A. baumannii & 13 & $1(7.69)$ & $0(0)$ & $0(0)$ & $3(23.08)$ \\
\hline P. aeruginosa & 10 & $0(0)$ & $0(0)$ & $0(0)$ & $1(10)$ \\
\hline A. Iwoffii & 01 & $0(0)$ & $0(0)$ & $0(0)$ & $0(0)$ \\
\hline S. maltophilia & 01 & $0(0)$ & $0(0)$ & $0(0)$ & $1(100)$ \\
\hline Total & 61 & $8(13.11)$ & $4(6.57)$ & $3(4.92)$ & $7(11.48)$ \\
\hline \multicolumn{6}{|r|}{ Table 3. $\boldsymbol{\beta}$ Lactamase Production in the Isolates of OSM } \\
\hline
\end{tabular}

Out of 115 cases of osteomyelitis, $98(85.22 \%)$ cases were of chronic osteomyelitis and $17(14.78 \%)$ were of acute osteomyelitis. Osteomyelitis was more common in males 79 $(68.70 \%)$ as compared to females $36(31.30 \%)$. However, the difference was not statistically significant ( $p>0.05)$ using pooled out data for up to 20 years, $21-40$ years, $>40$ years. It is observed that 21-30 years age group patients were affected the most $(26.96 \%)$. Long bones were maximally affected in osteomyelitis. Femur was the most common bone affected comprising $(60.87 \%)$, followed by tibia $(27.83 \%)$, humerus (5.22\%).

\section{DISCUSSION}

The present study was carried out in 115 clinically diagnosed patients of Osteomyelitis (OSM). In 101 samples, 116 organisms were isolated. And in 14 samples no organism was isolated. No growth can be attributed to the viral aetiology, parasites and anaerobes. The major systems of classification presently are Waldvogel classification ${ }^{9}$ and Cierny-Mader staging system. ${ }^{10}$ In the present study, AOSM was found to be more in the age group of 1-10 years of age group, whereas 
COSM was found more in 21-30 and 31-40 years of age group. In the present study, prevalence of OSM was found to be more in males $(68.70 \%)$ as compared to females $(31.30 \%)$ with Male to Female ratio was 2.2:1. Similar result was observed by Carvalho VC et al (2012) ${ }^{11}$ affecting $63.4 \%$ males and $36.6 \%$ females with OSM. Similar result was obtained by Waldvogel et al (1970) ${ }^{9}$ with Male to Female ratio was 2:1, Hassani U et al (2014) ${ }^{12}$ observed male predominance with Male to Female ratio was 1.95:1, and Wadekar MD et al (2014) ${ }^{13}$ observed Male to Female ratio was 2.7:1. While Izadi et al (2012) ${ }^{14}$ observed, prevalence of COSM was (81.7\%) in males and $(18.3 \%)$ in females, Ali $\mathrm{M}$ et al $(2014)^{15}$ noted incidence of OSM in males (84\%) and in females (16\%).

In the present study, bones involved in AOSM were femur (70.59\%), tibia (11.76\%), humerus (11.76\%). whereas in COSM were femur (59.18\%), tibia (30.61\%). Similarly, Ali M et al (2014) $)^{15}$ observed bones involved in COSM were femur (46\%), tibia (30\%), humerus (4\%). Wadekar MD et al $(2014)^{13}$ observed femur (48\%), tibia (23\%), humerus $(9 \%)$ and ulna (4\%). Whereas, Izadi et al (2012) ${ }^{14}$ found COSM mostly affected tibia (33\%), femur (27). Out of the 16 culture positive samples from AOSM, 20 organisms were isolated. $S$. aureus leads followed by $K$. pneumoniae and $A$. baumannii. Carvalho VC et al (2012) ${ }^{11}$ found A. baumannii (21.4\%), $P$. aeruginosa (19.8\%), K. pneumoniae (8.2\%) and E.coli (4.9\%) in AOSM. Mirnejad R et al (2008) ${ }^{16}$ found S. aureus (55.9\%), Klebsiella spp. (14.8\%), Coagulase negative Staphylococcus (7.4\%) Acinetobacter spp. (3.7\%) as cause of AOSM. Craigen MAC et al (1992) ${ }^{17}$ observed S. aureus (88.2\%), S. pyogenes (3.8\%) and E.coli (0.6\%) isolates in AOSM.

In the present study, out of the 85 culture positive samples from COSM, 96 organisms were isolated. Staphylococcus aureus 40 (41.67\%) was the most common isolated followed by K. pneumoniae and A. baumannii. Among the fungal agents, Candida albicans was isolated. In this study, $S$. aureus was the most predominant isolate as also seen in other studies such as Rahbar M et al (2010) ${ }^{18}$ as (26.3\%), Wadekar MD et al (2015) ${ }^{19}$ as (32.9\%), Izadi M et al (2012) ${ }^{14}$ as $48.9 \%$, Wirbel $\mathrm{R}$ et al $(2014)^{20}$ as $(74 \%)$. Ali $\mathrm{M}$ et al (2014) $^{15}$ observed S. aureus (58\%), Coagulase negative Staphylococcus (14\%). Thus, Staphylococcus aureus has been found to be the major etiological agent in our study, which is similar to other studies. Three cases in the present study were of sickle cell disease presenting with chronic osteomyelitis. Of which two cases yielded Salmonella Typhi from the pus aspirate and in one case Staphylococcus aureus was isolated. Thanni LOA et al (2006) ${ }^{21}$ isolated 304 cases of COSM with sickle cell disease, out of which 129 were $S$. Typhi and 82 were $S$. aureus.

All the isolates of $S$. aureus showed $100 \%$ sensitivity to Vancomycin, Amikacin, Netilmicin, Chloramphenicol and resistant to Penicillin G, Rifampicin. Ali M et al (2014) ${ }^{15}$ found S. aureus was $100 \%$ sensitive to Vancomycin, highly resistant to Cephalosporins, least sensitive to Ciprofloxacin, Amikacin and Gentamicin. Izadi et al (2012) ${ }^{14}$ found $S$. aureus was most sensitive to Vancomycin (97.7\%) and least sensitive to Penicillin (7\%). Wadekar MD et al (2014) ${ }^{13}$ observed that in Cosm S. aureus was (100\%) sensitive to Vancomycin, (97.1\%) to Linezolid and resistant to Ciprofloxacin, Erythromycin, Gentamicin, Clindamycin and Amikacin. Increasing resistance to Penicillin has been observed over the years. The studies in literature clearly show this resistance pattern. In the study done by Izadi et al (2012) ${ }^{14} 93 \%$ of the $S$. aureus were found resistant to penicillin.

In the present study, out of 48 isolates of $S$. aureus, $37.50 \%$ were MRSA, $6.25 \%$ were ICR, $14.58 \%$ were MRSA + ICR found. By E-strip method, $95.83 \%$ of the $S$. aureus isolates were sensitive and $4.17 \%$ were intermediate sensitive (IS) to Vancomycin. Ali M et al (2014) ${ }^{15}$ observed $42 \%$ of MRSA strains in COSM. Wadekar MD et al (2015) ${ }^{19}$ found $40 \%$ MRSA in COSM. Izadi M et al (2012) ${ }^{14}$ found $75 \%$ of MRSA in COSM. Whereas, Wirbel R et al $(2014)^{20}$ observed $10 \%$ of MRSA in COSM. The probable reason for this can be, the difference in location of samples and consequently difference in strains. ${ }^{14} \mathrm{~K}$. pneumoniae showed $100 \%$ resistant to Ampicillin, followed by Amoxyclav, Cefuroxime, Cefoxitin and Ceftazidime. Similarly, Ali M et al (2014) ${ }^{15}$ observed $K$. pneumoniae was $100 \%$ resistant to Ampicillin, followed by Gentamicin and Cefuroxime. 100\% sensitive to Imipenem followed by Amikacin, Ciprofloxacin, Cefotaxime and Ceftazidime. Whereas, Wadekar MD et al (2014) ${ }^{13}$ found $K$. pneumoniae was $100 \%$ resistant to Ampicillin, Gentamicin, followed by Cefotaxime, Amikacin and $78.5 \%$ sensitive to Imipenem.

A. baumannii were $100 \%$ sensitive to Polymyxin B (300), followed by Imipenem, Aztreonam and Amikacin. A. Iwoffii was sensitive to Gentamicin, Amikacin, Tobramycin, Ciprofloxacin and Polymyxin B (300). Carvalho VC et al (2012) ${ }^{11}$ observed that $A$. baumannii was sensitive to Imipenem (62\%), Gentamicin (54\%) and Amikacin (27\%). P. aeruginosa isolates were $90 \%$ sensitive to Imipenem, $60 \%$ to Ciprofloxacin, Piperacillin, Aztreonam and $40 \%$ sensitive to Piperacillin-Tazobactam, Amikacin, Gentamicin. Ali M et al (2014) ${ }^{15}$ reported that Pseudomonas aeruginosa, was $100 \%$ sensitive to Imipenem, $60 \%$ to Ceftazidime, $40 \%$ to Amikacin and Ciprofloxacin. Wadekar MD et al (2014) ${ }^{13}$ observed that Pseudomonas aeruginosa, was $76.40 \%$ sensitive to Imipenem, $58.80 \%$ to Amikacin.

In the present study, ESBL rates for $K$. pneumoniae isolates is $20 \%$, whereas Wadekar MD et al $(2015)^{19}$ observed that ESBL rates were $85.7 \%$ for $K$. pneumoniae isolates and $75 \%$ for Enterobacter isolates in their study. In this study we observed, 4 (6.57\%) AmpC producers, while AmpC production reported by Rawat et al (2013) ${ }^{22}$ was $20.8 \%$ and $36.6 \%$ by Haider et al (2014).23 All these were Inducible AmpC producers. However, Haider et al (2014) 23 have found only $21.7 \%$ Inducible AmpC producers and $78.3 \%$ non-inducible AmpC producers, which is an alarmingly high percentage of derepressed mutants.

Co-expression of different beta lactamase enzymes were found in the present study. There were three strains showing ESBL and AmpC co-production (4.92\%). Rawat et al (2013) ${ }^{22}$ have found that ESBL and AmpC were co-produced by 25\% isolates in their study. Out of 13 Acinetobacter spp. isolated, ESBL was found in 1 (7.69\%). Goel V et al (2013) ${ }^{24}$ found that $17.9 \%$ of $A$. baumannii to be ESBL producers. In our study we observed that $23.08 \%$ A. baumannii and $10 \%$ P. aeruginosa were MBL producers, whereas Goel V et al (2013), ${ }^{24}$ found that $48.72 \%$ A. baumannii and $53.85 \%$ P. aeruginosa were plasmid mediated MBL enzyme producing strains detected by Imipenem-EDTA disk method. OSM resulting from fungi is uncommon. ${ }^{25}$ In the present study, $2(1.72 \%)$ isolates of Candida albicans were isolated from cases of OSM. 


\section{CONCLUSIONS}

Osteomyelitis is found to be highest in third decade, with the males being predominantly affected. Acute osteomyelitis is predominantly seen in children, whereas chronic osteomyelitis in adults. Even though Staphylococcus aureus has always remained the most common etiological agent of osteomyelitis, increasing infections due to Gram negative bacilli and even poly-microbial infections are gaining importance. MRSA infection is known to increase postoperative complications. Introduction of MBL or carbapenemase production in Gram negative bacilli is a matter of great concern. Timely knowledge of aetiology and antimicrobial resistance pattern of osteomyelitis isolates can help in rational use of antibiotics and control of drug resistance.

\section{ACKNOWLEDGEMENT}

1. Many thanks to my Guide, HOD madam, teachers, seniors, technicians and colleagues of Microbiology department, IGGMC, Nagpur.

2. Thanks to the Resident doctors of Orthopedics department, IGGMC, Nagpur.

3. Thanks to the Dean of IGGMC, Nagpur for allowing me to conduct the study.

\section{REFERENCES}

[1] Lew DP, Waldvogel FA. Osteomyelitis. Lancet 2004;364 (9431):369-79.

[2] Romanò CL, Romanò $\mathrm{D}$, Logoluso $\mathrm{N}$, et al. Bone and joint infections in adults: a comprehensive classification proposal. Eur Orthop Traumatol 2011;1 (6):207-17.

[3] De Boeck H. Osteomyelitis and septic arthritis in children. Acta Ortho Belg 2005;71 (5):505-15.

[4] Maraga NF, Gomez MM, Rathore MH. Outpatient parenteral antimicrobial therapy in osteoarticular infections in children. J Paed Orthop 2002;22 (4):506-10.

[5] Krogstad P. Osteomyelitis and septic arthritis. In: Textbook of pediatric infectious diseases. $5^{\text {th }}$ edn. Philadelphia: WB Saunders 2004: p. 713-30.

[6] Collee JG, Dugaid JP, Fraser AG, et al. Laboratory strategy in the diagnosis of infective syndromes. In: Collee JG, Fraser AG, Marmion BP, et al. eds. Mackie and McCartney practical medical microbiology. 14th edn. Delhi: Churchill Livingstone 2012: p. 53-94.

[7] M100-S24 Performance standard for Antimicrobial Susceptibility Testing: Twenty-fourth informational supplement. Clinical and Laboratory Standard Institute. 2014;34 (1):1-230.

[8] Bauer AW, Kirby WMM, Sherris JC, et al. Antibiotic susceptibility testing by a standardized single disk method. Am J Clin Pathol 1966;45 (4):493-6.

[9] Waldvogel FA, Medoff G, Swartz MN. Osteomyelitis: a review of clinical features, therapeutic considerations and unusual aspects (second of three parts). N Engl J Med 1970;282 (5):260-6.
[10] Cierny G 3rd, Mader JT, Penninck JJ. A clinical staging system for adult osteomyelitis. Clin Orthop Relat Res 2003; (414):7-24.

[11] Carvalho VC, Oliveira PR, Dal-Paz K, et al. Gram-negative osteomyelitis: clinical and microbiological profile. Braz J Infect Dis 2012;16 (1):63-7.

[12] Hassani U, Jalgaonkar SV, Agrawal G. Aerobic microbiological profile of contigious focus osteomyelitis. Natl J Integr Res Med 2014;5 (3):102-7.

[13] Wadekar MD, Anuradha K, Venkatesha D. Chronic osteomyelitis: aetiology and antibiotic susceptibility pattern. Int J Recent Trends in Sci \& Technol 2014;9 (3):337-40.

[14] Izadi M, Zamani MM, Mousavi SA, et al. Is vancomycine still a choice for chronic osteomyelitis empirical therapy in Iran ? Iran Red Crescent Med J 2012;14 (12):782-6.

[15] Ali M, Kumari R. Evaluation of bacteriological profile of chronic osteomyelitis in a tertiary care hospital. Int J Sci Res 2014;3 (11):383-5.

[16] Mirnejad R, Fallahi S, Kiani J, et al. Epidemic assessment of bacterial agents in osteomyelitis and their antibiotic resistance pattern determination. J Biol Sci 2008;8 (2):478-81.

[17] Craigen MA, Watters J, Hackett JS. The changing epidemiology of osteomyelitis in children. J Bone Joint Surg Br 1992;74 (4):541-5.

[18] Rahbar M, Blackwell N, Yadgarinia D, et al. Etiology and drug resistance pattern of osteomyelitis associated with combat-related injuries in Iraqi patients. Shiraz E Med J 2010;11 (2):73-8.

[19] Wadekar MD, Naganath M, Venkatesha D. Detection of ESBL, MBL and MRSA among isolates of chronic osteomyelitis and their antibiogram. Int J Curr Microbiol Appl Sci 2015;4 (10):289-95.

[20] Wirbel R, Hermans K. Surgical treatment of chronic osteomyelitis in children admitted from developing countries. African J Paediatr Surg 2014;11 (4):297-303.

[21] Thanni LOA. Bacterial osteomyelitis in major sickling haemoglobinopathies: geographic difference in pathogen prevalence. Afr Health Sci 2006;6 (4):236-9.

[22] Rawat V, Singhai M, Verma PK. Detection of betalactamases and their co-existence by using various disc combination methods in clinical isolates of enterobacteriaceae and pseudomonas species. J Lab Physicians 2013;5 (1):21-5.

[23] Haider M, Rizvi M, Fatima N, et al. Necessity of detection of extended spectrum beta-lactamase, AmpC and metallo-beta-lactamases in Gram-negative bacteria isolated from clinical specimens. Muller J Med Sci Res 2014;5 (1):23-8.

[24] Goel V, Hogade SA, Karadesai SG. Prevalence of extended-spectrum beta-lactamases, AmpC beta lactamase, and metallo - beta - lactamase producing Pseudomonas aeruginosa and acinetobacter baumannii in an intensive care unit in a tertiary care hospital. J Sci Soc 2013;40 (1):28-31.

[25] Berbari EF, Steckelberg JM, Osmon DR. Osteomyelitis. In: Mandell GL, Bennett JE, Dolin R, eds. Principles and Practice of Infectious Diseases. $7^{\text {th }}$ edn. United States: Churchill Livingstone/ Elsevier 2010: p. 1457-74. 